\title{
T cell homeostatic proliferation elicits effective antitumor autoimmunity
}

\author{
Wolfgang Dummer, ${ }^{1}$ Andreas G. Niethammer, ${ }^{1}$ Roberto Baccala, ${ }^{1}$ Brian R. Lawson, ${ }^{1}$ \\ Norbert Wagner, ${ }^{2}$ Ralph A. Reisfeld, ${ }^{1}$ and Argyrios N. Theofilopoulos ${ }^{1}$
}

${ }^{1}$ Department of Immunology, The Scripps Research Institute, La Jolla, California, USA

${ }^{2}$ Department of Pediatrics, Klinikum Dortmund, Dortmund, Germany

\begin{abstract}
Development of tumor immunotherapies focuses on inducing autoimmune responses against tumorassociated self-antigens primarily encoded by normal, unmutated genes. We hypothesized that such responses could be elicited by $\mathrm{T}$ cell homeostatic proliferation in the periphery, involving expansion of $\mathrm{T}$ cells recognizing self-MHC/peptide ligands. Herein, we demonstrate that sublethally irradiated lymphopenic mice transfused with autologous or syngeneic $\mathrm{T}$ cells showed tumor growth inhibition when challenged with melanoma or colon carcinoma cells. Importantly, the antitumor response depended on homeostatic expansion of a polyclonal $\mathrm{T}$ cell population within lymph nodes. This response was effective even for established tumors, was characterized by $\mathrm{CD}^{+} \mathrm{T}$ cell-mediated tumorspecific cytotoxicity and IFN- $\gamma$ production, and was associated with long-term memory. The results indicate that concomitant induction of the physiologic processes of homeostatic $\mathrm{T}$ cell proliferation and tumor antigen presentation in lymph nodes triggers a beneficial antitumor autoimmune response.
\end{abstract}

J. Clin. Invest. 110:185-192 (2002). doi:10.1172/JCI200215175.

\section{Introduction}

Tumor-associated antigens are largely tissue-specific or differentiation antigens encoded by normal genes (1-4). This has been established primarily with melanoma wherein, with rare exceptions, the associated antigens are either (a) a class of lineage-specific melanosome membrane proteins also expressed by normal melanocytes, or (b) differentiation proteins expressed by melanoma cells and spermatozoa (5-7). Differentiation antigens are also involved in immunity to other tumors, including colon, breast, prostate, and pancreatic carcinomas $(6,8)$. These proteins, and corresponding peptides, are therefore true self-antigens, and the immune responses elicited against them are true autoimmune responses.

An organism uses several mechanisms to confer selftolerance and avoid harmful autoimmune responses, including negative selection in the thymus, deletion or anergy of peripheral $\mathrm{T}$ cells by incompetent antigen-presenting cells, such as immature dendritic cells (DCs), and immunosuppression by regulatory $\mathrm{T}$ cell subsets (8). Several of these tolerance mechanisms have been

Received for publication February 1, 2002, and accepted in revised form May 28, 2002.

Address correspondence to: Argyrios N. Theofilopoulos, Department of Immunology, The Scripps Research Institute, 10550 North Torrey Pines Rd/IMM3, La Jolla, California 92037, USA. Phone: (858) 784-8135; Fax: (858) 784-8361; E-mail: argyrio@scripps.edu.

Wolfgang Dummer and Andreas G. Niethammer contributed equally to this work.

Conflict of interest: No conflict of interest has been declared. Nonstandard abbreviations used: dendritic cells (DCs); lymph node (LN); carboxyfluorescein-diacetate-succinimidyl-ester (CFSE); recombination-activating gene-deficient (RAG-deficient). invoked to explain the absence or weak immune responses to tumor-associated antigens. Nevertheless, a variety of approaches have been used to circumvent peripheral tolerance and elicit $T$ cell responses against tumor self-molecules, particularly with melanoma, including vaccination with whole tumor cell extracts, recombinant vaccinia incorporating melanocyte-specific antigenic peptides, or cDNA-based vaccines encoding such epitopes $(1,6)$. DCs fused to tumor cells pulsed with peptides or transfected with RNA- or DNA-encoding tumor antigens have also been used $(9,10)$. However, although significant progress has been made, breaking tolerance for poorly antigenic self-peptides expressed by tumor cells remains a major challenge.

It is well-known that lymphopenia is followed by spontaneous expansion of the remaining $\mathrm{T}$ cells in the periphery to restore the original $\mathrm{T}$ cell pool size and maintain homeostasis (11). Recent studies have shown that lymphopenia-induced homeostatic proliferations of $\mathrm{CD}^{+}$and $\mathrm{CD}^{+} \mathrm{T}$ cells in the periphery are driven by recognition of self-MHC/peptide ligands similar to those that control their positive selection in the thymus (12-14). Thus, a certain degree of continuous autorecognition is a physiologic requirement to maintain $\mathrm{T}$ cell homeostasis. Since homeostatically proliferating $\mathrm{T}$ cells share some characteristics with effector/memory cells (12-14), we hypothesized that this self-MHC/peptide-driven proliferation could, under certain circumstances, be sufficient to induce autoimmune disease manifestations (15). Considering that self-molecules are the target of $\mathrm{T}$ cell antitumor responses, we now demonstrate that homeostatic $\mathrm{T}$ cell proliferation can lead to an autoimmune response against tumors that, in this case, is highly desirable and beneficial to the host. 


\section{Methods}

Mice. C57BL/6 (H2 ${ }^{\mathrm{b}}$, Thy 1.2 $\left.{ }^{+}\right)$and B6.PL $\left(\mathrm{H} 2^{\mathrm{b}}\right.$, Thy1.1 $\left.{ }^{+}\right)$ mice, 6-8 weeks of age, were obtained from the rodentbreeding colony at The Scripps Research Institute. B6. $\mathrm{Rag}^{-/-}$and B6.LT $\alpha^{-/-}$mice $\left(\mathrm{H} 2^{\mathrm{b}}\right.$, Thy $\left.1.2^{+}\right)$were purchased from The Jackson Laboratory (Bar Harbor, Maine, USA). C57BL/ 6 mice transgenic for the $2 \mathrm{C} \mathrm{T}$ cell receptor, which recognizes SIYRYYGL peptide in association with $\mathrm{MHC}$ class I $\mathrm{K}^{\mathrm{b}}$ molecule (16), were a gift from Jonathan Sprent (The Scripps Research Institute). B6. $\beta 7 / C D 62 L^{-/-}$mice were provided by Norbert Wagner (University of Cologne, Cologne, Germany).

Tumor cell injections. The B6-derived B78D14 murine melanoma (17) and the MC-38 colon cancer cell line (18) have been described. Mice were challenged subcutaneously in the left front flank with $5 \times 10^{5}$ melanoma cells or $10^{5}$ colon carcinoma cells and examined every other day. Tumor diameters were measured in two dimensions, and their volume was determined according to $1 / 2 \times$ length $\times$ width ${ }^{2}$.

Adoptive transfer of $T$ cells. Whole lymph node (LN) or purified $T$ cells were adoptively transferred by intravenous injection $\left(5 \times 10^{6}\right.$ or $\left.5 \times 10^{7}\right)$ into syngeneic recipients. Lymphopenia was induced by sublethal irradiation (600 cGy) of B6 mice 1 day before donor cell injection, while B6. Rag ${ }^{-1-}$ mice, lacking T and B cells, were used nonirradiated. For purification of $\mathrm{CD}^{+}$and $\mathrm{CD}^{+} \mathrm{T}$ cells, whole $\mathrm{LN}$ cell suspensions were stained with FITC-conjugated anti-CD4 or anti-CD8 Ab (PharMingen, La Jolla, California, USA) and sorted using a FACS Vantage cell sorter (Becton Dickinson Immunocytometry Systems, Mountain View, California, USA). A fraction of the cell populations was consistently screened by FACS analysis for expression of activation markers (CD44, CD25, CD69, CD45RB, CD62L) prior to injection. Lymph node T cells depleted of regulatory $\mathrm{CD} 25^{+}$cells were prepared using an AutoMACS magnetic cell sorter (Miltenyi Biotec, Bergisch Gladbach, Germany), according to the manufacturer's protocols and as detailed by Gavin et al. (19); CD25- populations were $98 \%$ pure.

In vivo proliferation and FACS analyses. To establish homeostatic $\mathrm{T}$ cell proliferation of different populations in various hosts, donor cells were labeled with the intracellular fluorescent dye carboxyfluorescein-diacetate-succinimidyl-ester (CFSE; Molecular Probes Inc., Eugene, Oregon, USA) prior to adoptive transfer, as we described previously (20). Donor cell proliferation was measured by stepwise reduction of CFSE intensity using FACS analysis on day 7; distinct peaks represent the number of cell divisions in LN and spleen cell suspensions. Identification of donor $\mathrm{T}$ cells was accomplished by staining with a biotinylated anti-Thy1.1 or anti-Thy1.2 Ab (PharMingen), followed by Cy5-conjugated streptavidin (Jackson ImmunoResearch Laboratories Inc., West Grove, Pennsylvania, USA).

CFSE levels were measured on gated Thy $1.1^{+} \mathrm{CD} 4^{+}$or Thy $1.1^{+} \mathrm{CD}^{-}\left(\mathrm{CD}^{+}\right)$donor cells. The numbers of Thy $1.1^{+}$donor cells in the recipients' secondary lymphoid organs (LNs and spleen) were calculated using the percentage of distribution of cells in FACS dot plots combined with total cell counts from the respective single cell suspensions. CFSE histograms were used to calculate the percentages of proliferating donor cells that had undergone one or more cell divisions.

Cytotoxicity and IFN- $\gamma$ assays. To assess tumor-specific cytotoxicity, effector splenocytes were obtained at 10 days after challenge from B6 mice that were sublethally-irradiated, transfused with $5 \times 10^{6}$ syngeneic lymph node cells, and injected with B78D14 melanoma cells. The splenocytes were cultured at $37^{\circ} \mathrm{C}$ for 48 hours with either irradiated B78D 14 melanoma cells or the irrelevant MC-38 colon carcinoma cells at 100:1 effector-to-target cell ratios. Subsequently, cytotoxicity was assessed at 200:1, 100:1, and 50:1 effector-to-target cell ratios using a standard 4-hour ${ }^{51} \mathrm{Cr}$-release assay. The percentage of target cell lysis was calculated as follows: [(experimental release cpm) - (spontaneous release cpm) $] /[$ total release cpm) - (spontaneous release cpm) $\times 100$.

For IFN- $\gamma$ determination, effector splenocytes were harvested at day 35 after challenge from sublethally irradiated syngeneic LN cell-transfused $\left(5 \times 10^{6}\right)$ and B78D14 melanoma cell-injected B6 mice. Effector splenocytes were cultured as noted above with irradiated relevant (B78D14) or irrelevant (MC-38) tumor cells at 100:1 effector-to-target cell ratios and supernatant collected at 72 hours. IFN- $\gamma$ was measured using a commercial kit (OptEIA mouse; PharMingen), according to manufacturer's instructions, and concentrations were expressed as picograms per milliliter relative to the standard curve.

Immunohistochemistry. Tumor specimens and regional LNs were embedded into OCT compound (Tissue-Tek) and snap-frozen in liquid nitrogen. Immunohistochemistry was performed for $\mathrm{CD}^{+}, \mathrm{CD}^{+}, \mathrm{CD}^{+} 6^{+}$, and Thy $1.1^{+}$cells according to standard procedures.

Statistics. Statistical significance was determined by the two-tailed Student $t$ test, and $P$ values less than 0.05 were considered significant.

\section{Results}

Growth of melanoma in lymphopenic hosts transfused with syngeneic $T$ cells. Sublethal irradiation (600 cGy) is a common means of inducing lymphopenia in mice to study homeostatic proliferation after adoptive transfer of syngeneic $T$ cells. In this system, donor T cells are differentiated from recipient cells by allelic markers (i.e., Thy 1.1/1.2 or CD45) and/or intracellular dyes (i.e., CFSE) (13). We used these approaches to determine whether homeostatic $\mathrm{T}$ cell expansion is associated with protective antitumor autoimmunity.

Nonirradiated and sublethally irradiated C57BL/6 (B6) mice were challenged 24 hours after irradiation with a subcutaneous injection of $5 \times 10^{5}$ B78D14 melanoma cells, and tumor growth was assessed. As expected, at day 52 after challenge, all control nonirradiated mice had developed large tumors, with a mean 


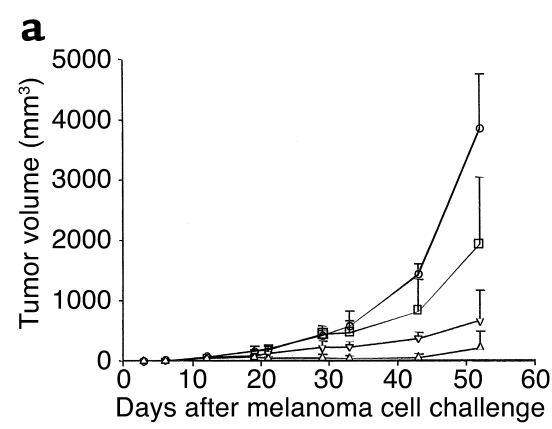

b

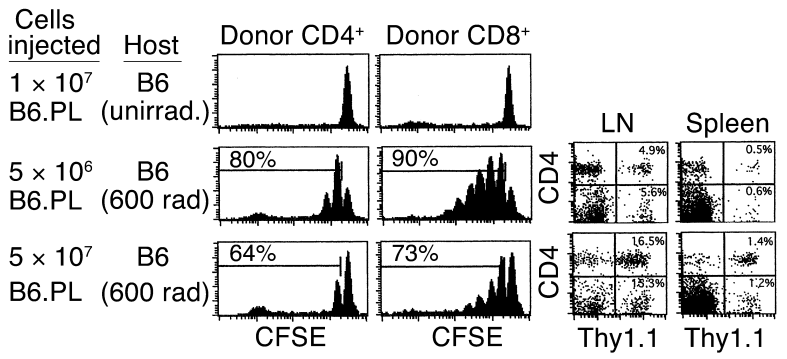

C

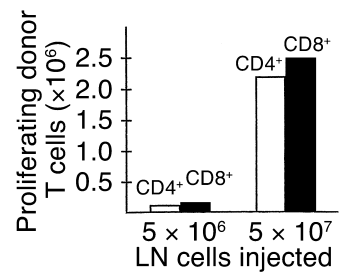

\section{Figure 1}

Inhibition of melanoma growth by homeostatic T cell proliferation. (a) Nonirradiated (circles) or sublethally irradiated (squares) C57BL/6 mice were challenged subcutaneously with $5 \times 10^{5}$ B78D14 melanoma cells. Groups of irradiated mice were transfused with $5 \times 10^{6}$ (inverted triangles) or $5 \times 10^{7}$ (triangles) syngeneic B6.PL LN cells. Means and standard deviation of tumor growth are indicated (all groups, $n=7)$. (b) Proliferation profiles of gated Thy $1.1^{+} \mathrm{CD} 4^{+}$and Thy $1.1^{+} \mathrm{CD} 4^{-}\left(\mathrm{CD} 8^{+}\right)$cells in secondary lymphoid organs shown as CFSE histograms. Upper panel: nonirradiated C57BL/ 6 mice (Thy1.2+) transfused with $10^{7} \mathrm{~B} 6 . \mathrm{PL}\left(\right.$ Thy $1.1^{+}$) cells; middle panel: C57BL/6 mice irradiated and transfused with $5 \times 10^{6} \mathrm{~B} 6 . \mathrm{PL} \mathrm{LN}$ cells; lower panel: C57BL/ 6 mice irradiated and transfused with $5 \times 10^{7} \mathrm{~B} 6 . \mathrm{PL} \mathrm{LN}$ cells. FACS dot plots of host lymphoid organs are depicted to the right. (c) Total number of proliferating (one or more cell divisions) donor $\mathrm{CD}^{+}$and $\mathrm{CD} 8^{+}$cells in $\mathrm{C} 57 \mathrm{BL} / 6$ recipients of $5 \times 10^{6}$ or $5 \times 10^{7} \mathrm{~B} 6$. PL LN cells calculated from data presented in $\mathbf{b}$.

size of $3,837 \pm 917 \mathrm{~mm}^{3}$ (Figure 1a). In contrast, the mean tumor size for the sublethally irradiated recipients was $1,924 \pm 111 \mathrm{~mm}^{3}(P<0.05)$.

We then examined whether the antitumor effect after irradiation alone could be enhanced by adoptive transfer of syngeneic $\mathrm{T}$ cells, since homeostatic $\mathrm{T}$ cell expansion subsequent to irradiation only is protracted, and the $\mathrm{T}$ cells do not appear to be fully functional. To this end, B6 mice (Thy $1.2^{+}$) were sublethally irradiated, transfused 24 hours later with either $5 \times 10^{6}$ or $5 \times 10^{7}$ syngeneic B6.PL (Thy1.1 $1^{+}$LN cells, and challenged with $5 \times 10^{5}$ B78D14 melanoma cells. Transfusion of the smaller cell dose led to a further reduction in tumor size compared with nontransfused sublethally irradiated mice $\left(655 \pm 496 \mathrm{~mm}^{3}\right.$ vs. $1,924 \pm 111$ $\mathrm{mm}^{3}, P<0.02$; Figure 1a). Tumor growth inhibition was even more evident in mice transfused with $5 \times 10^{7}$
LN cells, with tumors reduced to almost undetectable levels in four of seven animals at day 30 after challenge and an average tumor size of $210 \pm 271 \mathrm{~mm}^{3}$ at day 52 $(P<0.01$, Figure 1a).

Proliferation characteristics of $5 \times 10^{6}$ or $5 \times 10^{7}$ CFSE-labeled syngeneic B6.PL LN cells transfused into sublethally irradiated $\mathrm{B} 6$ recipients at day 7 after transfer are shown in Figure 1b. The smaller population of $T$ cells undergoes more divisions, as defined by the number and size of peaks depicting diminishing CFSE intensity. The higher number of transferred donor cells proliferated more slowly, consistent with the idea of less available space (20). Yet, when the percentage of donor (Thy $\left.1.1^{+}\right) \mathrm{T}$ cells that had entered one or more divisions was analyzed in host $\mathrm{LN}$ and spleen cell suspensions on day 7, we calculated that approximately ten times more donor cells proliferated with the larger than with the
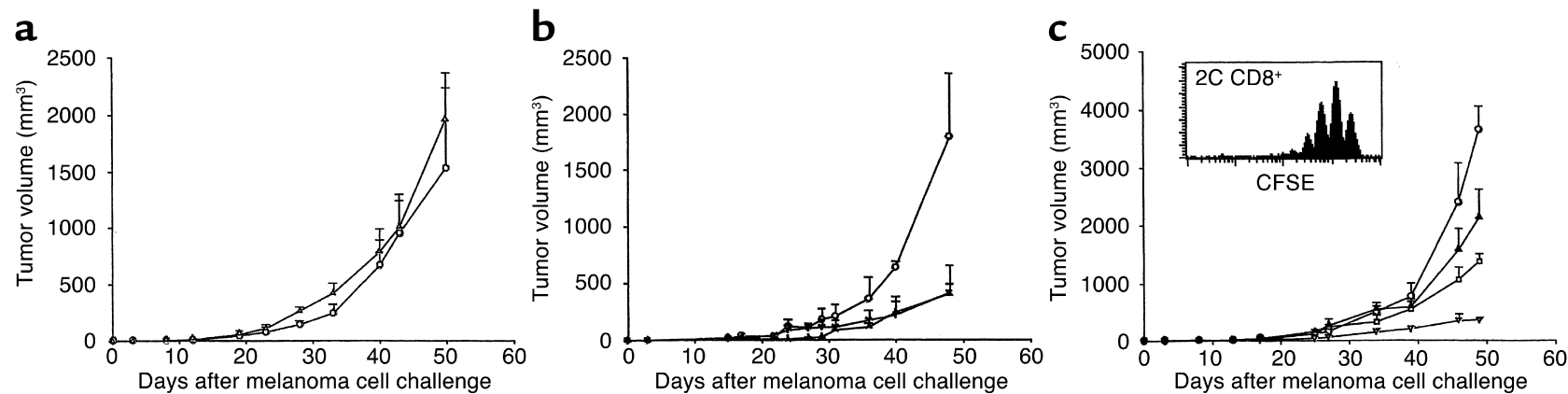

Figure 2

Polyclonal homeostatic expansion of $\mathrm{CD} 8^{+} \mathrm{T}$ cells is sufficient for melanoma growth inhibition. (a) Nonirradiated (circles) and irradiated (triangles) B6.RAG ${ }^{-/-}$mice challenged with melanoma cells show no difference in tumor growth ( $n=5$ for each group). (b) Transfusion of $5 \times 10^{6}$ syngeneic B6.LN cells to nonirradiated $B 6 . R A G^{-1-}$ mice leads to significant melanoma growth inhibition (triangles) compared with nonirradiated, nontransfused controls (circles). Adoptive transfer of purified C57BL/ $6 \mathrm{CD} 8{ }^{+} \mathrm{T}$ cells is sufficient to exert an antitumor effect (squares) equal to that of whole LN cells (all groups, $n=7$ ). (c) Adoptive transfer of $5 \times 10^{6} 2 \mathrm{C} \mathrm{CD8} 8^{+}$TCR transgenic cells into irradiated B6 hosts (triangles) did not enhance the antitumor effect achieved by irradiation alone (squares), despite the ability of $2 \mathrm{C}$ cells to undergo homeostatic proliferation (inset). Controls included nonirradiated, nontransfused C57BL/ 6 mice (circles) and irradiated, transfused $\left(5 \times 10^{6} \mathrm{~B} 6 \mathrm{LN}\right.$ cells) C57BL/ 6 mice (inverted triangles) (all groups, $\left.n=6\right)$. 

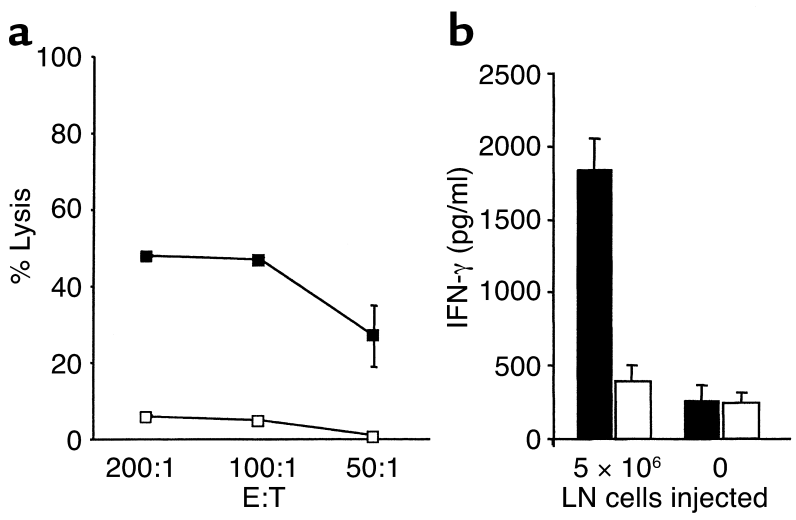

smaller dose of transfused cells (Figure 1c), thereby explaining the stronger antitumor effect. Nevertheless, at 10 weeks after transfer, the irradiated recipients yielded total LN and spleen cell counts of 4.5 to $5.5 \times 10^{7}$ and 12 to $14 \times 10^{7}$, respectively, comparable to unmanipulated mice, indicating full $\mathrm{T}$ cell reconstitution.

To determine whether the incomplete tumor inhibition in some animals might have been caused by the presence of regulatory/suppressor T cells in the lymphocyte inocula, separate groups of sublethally irradiated B6 mice were transfused with either $5 \times 10^{6}$ total syngeneic LN cells or a similar number of LN cells depleted of the $\mathrm{CD} 25^{+}$regulatory/suppressor $\mathrm{T}$ cell subset and challenged with melanoma cells $(n=3-6$ mice/group). At day 29 after transfer, tumor inhibition was incomplete in both instances. This issue needs to be explored further.

The antitumor effect observed was mediated by polyclonal homeostatic $T$ cell expansion and not by a nonspecific effect of irradiation or lymphocyte transfusion. Three lines of evidence support this conclusion. First, tumor growth was unimpeded in nonirradiated mice transfused with $5 \times 10^{6}$ or $5 \times 10^{7}$ syngeneic LN cells (not shown) that showed no measurable $\mathrm{T}$ cell proliferation in immunocompetent hosts (Figure 1b). Second, recombination-activating gene-deficient (RAG-deficient) B6 mice lacking T and B cells, irradiated or not, revealed identical tumor growth (Figure 2a). Third, compared with irradiation alone, tumor growth was not further impaired in mice irradiated and transfused with a $\mathrm{T}$ cell clone (2C) specific for a synthetic peptide (SIYRYYGL) presented in the context of MHC class I $\mathrm{K}^{\mathrm{b}}$ molecules (16), although these 2C cells did undergo efficient homeostatic expansion (Figure 2c). The data from the latter experiment also suggest that preexisting $\mathrm{T}$ cells recognizing tumor antigens within the normal repertoire mediate the antitumor effect, since the transgenic $\mathrm{CD}^{+}$cells with specificity for an irrelevant peptide are ineffective despite their excessive homeostatic expansion.

Tumor rejection is mediated by homeostatic expansion of $C D 8^{+} T$ cells. Protective autoimmunity against

\section{Figure 3}

Specific recognition of tumors induced by homeostatic proliferation. (a) Sublethally irradiated C57BL/ 6 mice were transfused with $5 \times 10^{6}$ LN cells prior to challenge with B78D14 melanoma cells $(n=4)$. Ten days after challenge, splenocytes were coincubated with either irradiated B78D14 melanoma cells (filled squares) or irradiated MC-38 colon carcinoma cells (open squares) for 48 hours; a standard 4-hour ${ }^{51} \mathrm{Cr}$-release assay was performed at three effector-to-target cell ratios. Results indicate percentage of specific lysis from a pool of four mice assessed in triplicate. (b) Sublethally irradiated C57BL/ 6 mice were transfused with $5 \times 10^{6} \mathrm{LN}$ cells prior to challenge with B78D14 melanoma cells. Thirty-five days after challenge, splenocytes were cultured for 72 hours with either B78D14 melanoma cells (black bars) or MC-38 colon carcinoma cells (white bars). Similarly cultured control lymphocytes were derived from nonirradiated, nontransfused, and melanoma-challenged mice. Results indicate IFN- $\gamma$ levels in the supernatants from a pool of four mice assessed in quadruplicate.

melanoma tumors achieved by various vaccination protocols reportedly requires $\mathrm{CD} 8, \mathrm{CD} 4$, or both $\mathrm{T}$ cell subsets (21-24). To evaluate which $\mathrm{T}$ cell subset is primarily responsible for tumor inhibition in our system, we examined tumor growth in $R A G^{-/-}$B6 mice transfused with $5 \times 10^{6} \mathrm{LN}$ cells, $5 \times 10^{6}$ FACS-sorted $\mathrm{CD}^{+}$ syngeneic cells, or $5 \times 10^{6}$ FACS-sorted $\mathrm{CD}^{+}$syngeneic cells and challenged with $5 \times 10^{5}$ B78D14 melanoma cells. Tumor growth was equally inhibited by total $\mathrm{LN}$ or $\mathrm{CD}^{+}$cells alone $(99.8 \%$ purity), indicating that tumor rejection was induced by the homeostatic expansion of $\mathrm{CD}^{+} \mathrm{T}$ cells (Figure $2 \mathrm{~b}$ ). Transfer of $\mathrm{CD}^{+}$cells led to colitis, weight loss, and death of the $R A G^{-/-}$recipients after about 3 weeks, consistent with previous reports $(25,26)$. Nevertheless, the results suggest that the expanded $\mathrm{CD}^{+}$cells, presumably due to the high MHC class I affinity of the immunogen(s) (27) or high precursor frequency (28), do not require $\mathrm{CD}^{+} \mathrm{T}$ cell help to exert their antitumor effect.

Tumor-specific cytotoxicity and IFN- $\gamma$ production. In vitro cytotoxicity and IFN- $\gamma$ production by the homeostatically expanded $\mathrm{T}$ cells in mice challenged with melanoma cells were assessed to determine whether the induced autoimmune response was tumor specific. A strong cytotoxic response was elicited against the relevant B78D14 cells, whereas lysis of the irrelevant MC-38 colon carcinoma cells was marginal, even at the high effector-to-target cell ratios (Figure $3 a, P<0.01$ ). Similarly, the expanded $T$ cells produced significantly higher amounts of IFN- $\gamma$ upon incubation with the relevant B78D14 cells than upon incubation with the irrelevant MC-38 colon carcinoma cells (Figure 3b, $P<0.01)$. Lymphocytes from nonirradiated, nontransfused mice challenged with melanoma cells produced minimal levels of IFN- $\gamma$ when cultured with B78D14 cells or MC-38 cells.

Tumor infiltration by donor T cells. Sublethally irradiated B6 mice (Thy $1.2^{+}$) were transfused with $5 \times 10^{6}$ CFSE-labeled B6.PL (Thy1.1+) LN cells, challenged with melanoma cells, and, 4 weeks later, the division characteristics of the infused cells in secondary lymphoid organs and tumor infiltrates were assessed. As 
expected, donor T cells in spleen and LNs divided rapidly, as indicated by the large population seen at the extreme left of the histograms (Figure 4a). This fast proliferation was more prominent in $\mathrm{CD}^{+}$than in $\mathrm{CD}^{+}$tumor-infiltrating donor $\mathrm{T}$ cells. These results indicate that the infused $\mathrm{CD}^{+} \mathrm{T}$ cells had either divided more extensively within the tumor or homed preferentially to the tumor subsequent to their rapid expansion in secondary lymphoid organs.

Immunohistochemical analyses showed that $\mathrm{T}$ cell infiltrates in and around the regressing tumors contained primarily B6.PL donor $\mathrm{T}$ cells (Thy $1.1^{+}$) (Figure $4, \mathrm{~b}-\mathrm{g})$. Minimal $\mathrm{T}$ cell infiltrates were noted in the large tumor masses of control nonirradiated, nonlymphopenic B6 mice regardless of whether or not they had been transfused with B6.PL cells. Both $\mathrm{CD} 8^{+}$and $\mathrm{CD}^{+} \mathrm{T}$ cells infiltrated residual tumors, while far fewer such cells were detected in the large tumors of nonirradiated mice. Staining for CD56 revealed a negligible number of NK cells in all instances.

Homeostatic proliferation must occur in the regional LNs to induce the antitumor effect. We performed two types of experiments to examine whether homeostatic $\mathrm{T}$ cell expansion itself was sufficient to induce the antitumor response, or whether the tumor antigens must be presented to proliferating $\mathrm{T}$ cells in the relevant regional LNs. First, normal B6 mice or B6 mice deleted of the lymphotoxin- $\alpha$ gene $\left(L T \alpha^{-/}\right)$, which lack LNs and show disorganization of the splenic white pulp $(29,30)$, were sublethally irradiated, transfused with $5 \times 10^{6}$ B6 LN cells, and subsequently challenged with melanoma cells. Tumor growth inhibition was more efficient in control normal B6 mice than in LT $\alpha$-deficient mice (Figure 5a). However, separate experiments with CFSE-stained LN cells showed proliferation of transferred $\mathrm{CD}^{+} \mathrm{T}$ cells in the spleen of sublethally irradiated $\mathrm{LTO}^{-/-}$mice (Figure 5a, inset). Second, sublethally irradiated B6 mice were challenged with melanoma cells and transfused with $5 \times 10^{6}$ spleen cells from either normal B6 or B6 mice deficient for both the $\beta 7$ integrin and the CD62L (L-selectin) genes. T cells from such double-deficient mice lack the ability to enter peripheral LNs or Peyer's patches (31). Compared with irradiation only control mice, there was enhanced inhibition of tumor growth upon infusion of normal $\mathrm{T}$ cells, but not of $\beta 7 / C D 62 L^{-/-} \mathrm{T}$ cells (Figure $5 \mathrm{~b}$ ); yet CFSE-stained $\mathrm{T}$ cells of $\beta 7 / \mathrm{CD} 62 \mathrm{~L}$-deficient mice divided as well as normal $\mathrm{T}$ cells in the spleen of lymphopenic recipients (Figure $5 \mathrm{~b}$, inset). The combined findings suggest that homeostatically proliferating $\mathrm{T}$ cells must encounter the tumor antigens in regional LNs to be recruited for antitumor effector function.

Vitiligo as a manifestation of antimelanoma autoimmunity. In several irradiated mice transfused with LN cells and challenged with melanoma cells (three of four and four of seven with $5 \times 10^{6}$ or $5 \times 10^{7}$ transfused cells, respectively), we observed depigmentation of regenerated hair a
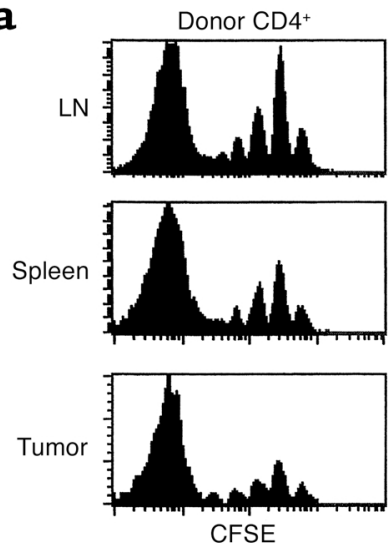
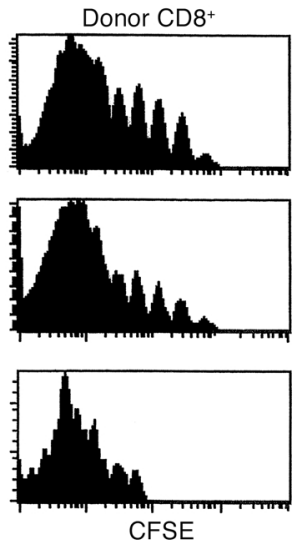

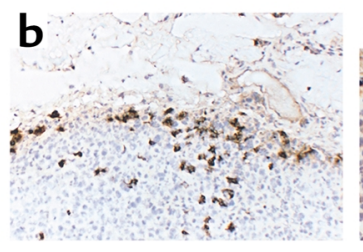

d

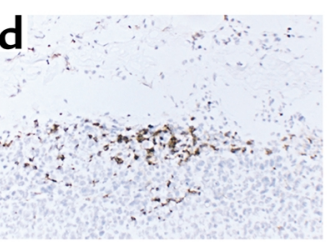

$\mathbf{f}$

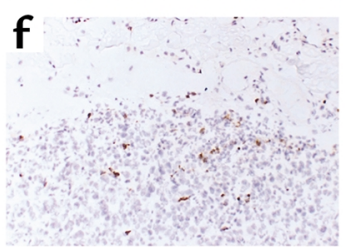

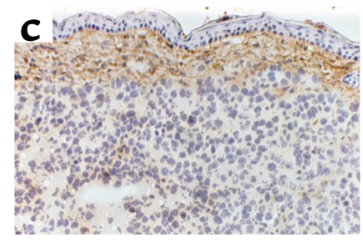
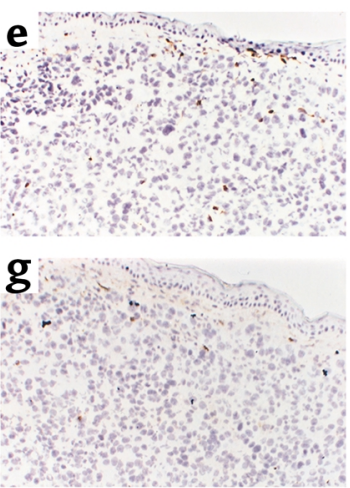

\section{Figure 4}

Proliferation and immunohistochemistry of tumor-infiltrating T cells. (a) Groups of C57BL/ 6 mice (Thy $1.2^{+}$) were sublethally irradiated, challenged with melanoma cells, and transfused with $5 \times 10^{6} \mathrm{CFSE}$-labeled syngeneic B6.PL (Thy1.1 $\left.{ }^{+}\right) \mathrm{LN}$ cells. Four weeks later, single cell suspensions of host $\mathrm{LN}$, spleen, and tumor tissue were analyzed for division characteristics by flow cytometry of gated Thy $1.1^{+} \mathrm{CD} 4^{+}$and Thy 1.1 ${ }^{+} \mathrm{CD} 4^{-}\left(\mathrm{CD} 8^{+}\right)$donor cells. (b-g) Groups of C57BL/ 6 mice were untreated or sublethally irradiated and challenged with melanoma cells. The irradiated mice were then transfused with $5 \times 10^{7}$ syngeneic B6.PL LN cells. Immunohistochemistry of residual tumors in transfused $(\mathbf{b}, \mathbf{d}, \mathbf{f})$ mice compared with large tumors of untreated controls $(\mathbf{c}, \mathbf{e}, \mathbf{g})$ is shown at day 60. Staining with an anti-Thy1.1 Ab (OX-7) reveals donor cells in the regressed tumor tissue of the LN cell-transfused irradiated host (b), but not in the progressing melanoma tumor of transfused, nonirradiated animals (c). Staining for $\mathrm{CD}^{+}$cells demonstrates considerable infiltration in the irradiated reconstituted group (d), but minor infiltration in the nonirradiated, nonreconstituted mice (e). Similarly, CD8 ${ }^{+}$cells are found in higher numbers in irradiated and transfused $(\mathbf{f})$ than in nonirradiated, nontransfused $(\mathbf{g})$ mice. Staining for CD56 did not reveal appearance of NK cells (not shown). 


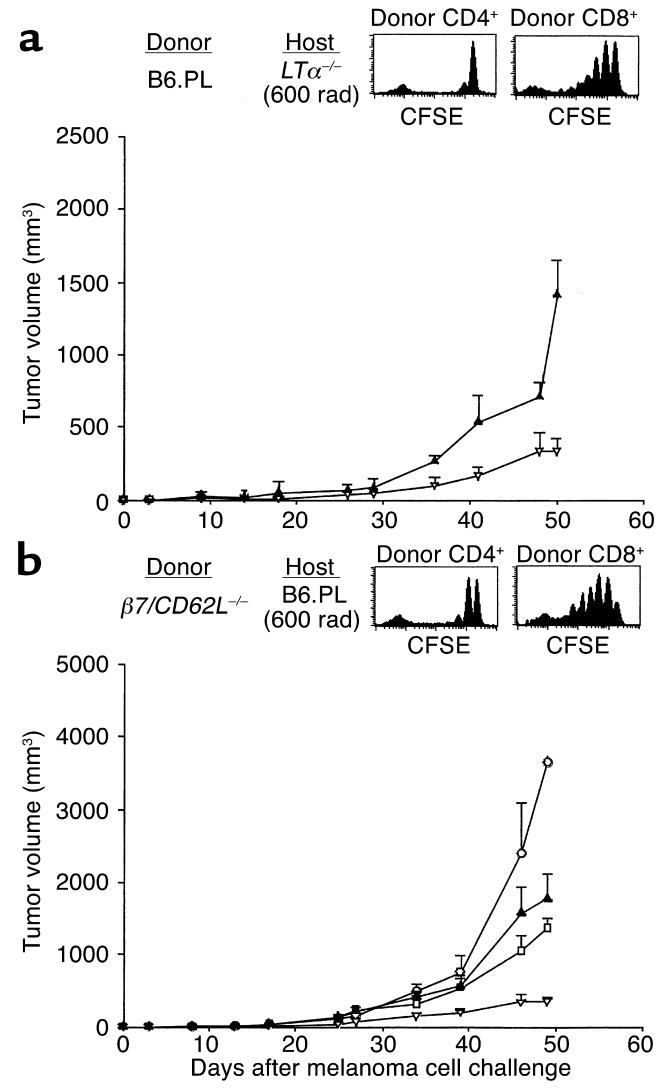

just above the depilated melanoma cell injection site. Such depigmentation was not seen in nonirradiated B6 mice challenged with melanoma cells and transfused with an equal number of $\mathrm{LN}$ cells. In all sublethally irradiated mice, whether injected with melanoma cells and/or transfused with syngeneic cells, there was also a diffuse mild hair depigmentation, particularly on the thorax and abdomen. No other macroscopic evidence of autoimmunity was noted in the sublethally irradiated mice transfused with LN cells and challenged with melanoma cells.

Long-term specific antitumor immunity and memory. Mice that had rejected melanoma cells completely were rechallenged at day 80 and day 200 with the same melanoma cells (B78D14) and the MC-38 colon carcinoma cells on the flank opposite the original injection. All animals rejected the melanoma cells, but not the colon carcinoma cells, verifying that rejection of melanoma cells during homeostatic $\mathrm{T}$ cell expansion induced specific antimelanoma memory. Ongoing observation of the rechallenged mice revealed no melanoma tumor growth even at day 300 after initial rejection.

Effect of homeostatic $T$ cell expansion on established melanoma tumors. We also addressed whether homeostatic $\mathrm{T}$ cell expansion can induce not only protective, but also therapeutic, antitumor autoimmunity. Thus, sublethally irradiated B6 mice were challenged with melanoma cells and, when tumors had reached a size of approximately $10 \mathrm{~mm}^{3}$, they were transfused with $5 \times 10^{7} \mathrm{~B} 6 \mathrm{LN}$ cells. At day 52 , tumor growth was

\section{Figure 5}

T cells need to home to the regional $\mathrm{LN}$ to mediate tumor growth inhibition. (a) Melanoma growth inhibition is less efficient in sublethally irradiated, LN cell-transfused LT $\alpha$-deficient B6 mice (triangles) than in $\mathrm{LT}^{+/+}$mice (inverted triangles) (both groups $n=6$ ). Homeostatic proliferation characteristics of CFSE-labeled B6.PL $\left(\right.$ Thy $\left.1.1^{+}\right) \mathrm{CD}^{+}$and $\mathrm{CD}^{+} \mathrm{T}$ cells in the spleen of an irradiated B6.LT $\alpha^{-/}$host is intact, as measured by flow cytometry on day 7 after transfer (inset). (b) Adoptive transfer of $\beta 7 / C D 62 L^{-/-}$spleen cells into irradiated C57BL/ 6 mice (triangles) does not lead to enhanced tumor growth inhibition compared with irradiated, nontransfused C57BL/ 6 controls (squares). Homeostatic proliferation of CFSE-labeled B6. $\beta 7 / \mathrm{CD} 6 \mathrm{~L}^{-/-}\left(\right.$Thy $\left.1.2^{+}\right) \mathrm{CD}^{+}$and $\mathrm{CD} 8^{+} \mathrm{T}$ cells in the spleen of an irradiated B6.PL host $\left(\right.$ Thy $\left.1.1^{+}\right)$is intact, as measured by flow cytometry on day 7 after transfer (inset). Controls included nonirradiated, nontransfused (circles), and irradiated C57BL/ 6 mice transfused with $5 \times 10^{6} \mathrm{~B} 6 \mathrm{LN}$ cells (inverted triangles).

significantly inhibited compared with irradiated and tumor cell-challenged control mice $\left(448 \pm 107 \mathrm{~mm}^{3}\right.$ vs. $\left.1,364 \pm 141 \mathrm{~mm}^{3}, P<0.01\right)$. These findings suggest that homeostatic $\mathrm{T}$ cell expansion can affect established tumors.

Effect of homeostatic $T$ cell expansion on colon carcinoma. To validate the applicability of protective antitumor autoimmunity by homeostatic $\mathrm{T}$ cell expansion to other tumors, we subcutaneously challenged sublethally irradiated $\mathrm{B} 6$ mice with $10^{5} \mathrm{MC}-38$ syngeneic colon carcinoma cells subsequent to reconstitution with a high number of autologous cells $\left(5 \times 10^{7} \mathrm{LN}\right.$ cells). Growth of this rapidly progressive tumor was severely impaired compared with control, nonirradiated, nontransfused mice (Figure 6). Unlike melanoma, however, all mice eventually developed colon carcinoma, albeit much slower than controls.

\section{Discussion}

Most tumor-associated antigens have been identified as normal gene products that are overexpressed, preferentially expressed, or reexpressed in cancer cells (1-8).

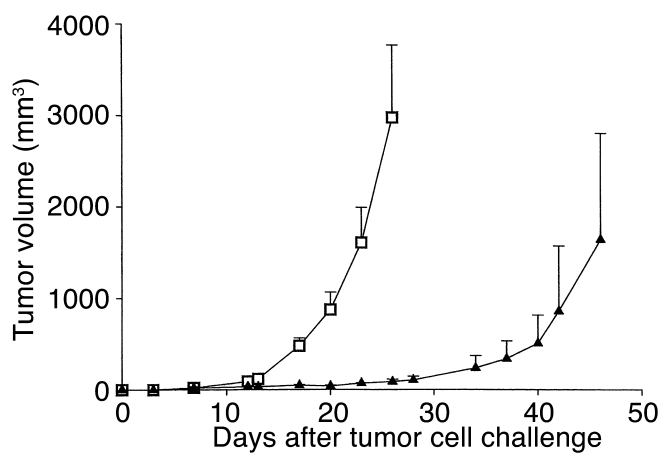

Figure 6

Effect of homeostatic T cell expansion on growth of a colon carcinoma cell line. Sublethally irradiated C57BL/ 6 mice were challenged subcutaneously with $10^{5} \mathrm{MC}-38$ colon carcinoma cells, and half of the mice were transfused with $5 \times 10^{7} \mathrm{~B} 6 \mathrm{LN}$ cells. Tumor growth was inhibited in the transfused (triangles) compared with the nontransfused (squares) mice (both groups, $n=6$ ). 
This has resulted in a new paradigm in tumor immunotherapy that has redirected efforts to break tolerance and induce autoimmune responses against such antigens $(1,3,5-8)$. Here, we demonstrate that lymphopenia-induced homeostatic $\mathrm{T}$ cell proliferation is a physiologic process by which effective antitumor autoimmunity can be elicited.

This investigation is based on the recent demonstration that self-MHC/peptide recognition is important for $\mathrm{T}$ cells to undergo homeostatic proliferation, a process that maintains the near constant size of the peripheral $\mathrm{T}$ cell pool (12-14). Homeostatic adjustment of $T$ cell numbers is necessary after lymphopenia has been induced by a variety of insults (infection, irradiation, or cytotoxic drugs), is polyclonal, and occurs without deliberate immunization.

An important question is whether T cells undergoing homeostatic proliferation can acquire effector function and thus cause autoimmunity. Several studies have shown that $\mathrm{T}$ cells driven to homeostatic proliferation acquired several, but not all, of the activation/memory phenotype markers associated with responses to foreign antigen (12-14, 32). Moreover, TCR-transgenic $\mathrm{CD}^{+}$cells that had undergone homeostatic proliferation were shown to kill target cells in vivo in a peptideand TCR-dependent manner, and to express IFN- $\gamma$ after stimulation with anti-CD3 $(33,34)$. Similarly, even polyclonal $\mathrm{CD}^{+}$cells that had undergone homeostatic proliferation rapidly secreted IFN- $\gamma$ after in vitro anti-CD3 stimulation and killed ConA-coated syngeneic targets; control naive $\mathrm{CD}^{+}$cells were devoid of such activities (34). We demonstrate here that polyclonal homeostatic proliferation leads to in vivo effector function against melanoma and, to some extent, colon carcinoma cells. Interestingly, this antimelanoma response was associated with vitiligo, also seen in other types of melanoma immunotherapies and considered to reflect therapeutic efficacy $(5,23)$. Our findings establish a fascinating link between self-recognition as a requirement for homeostatic $\mathrm{T}$ cell proliferation, antitumor responses, and autoimmune disease.

To undergo homeostatic expansion, $\mathrm{T}$ cells must enter certain areas in secondary lymphoid organs, such as periarteriolar lymphocyte sheaths in spleen and paracortex in LNs (20). Inhibition of tumor growth was much less evident when normal T cells were transfused into LT $\alpha$-deficient mice than into $\mathrm{LT}^{+/+}$mice. LT $\alpha$-deficient mice lack LNs and have variable degrees of disorganized splenic architecture $(29,30)$. More importantly, transfusion of $\beta 7 / C D 62 \mathrm{~L}$-deficient $\mathrm{T}$ cells, which do not home into LNs (31), but home and proliferate efficiently in the spleen, did not enhance the antitumor response seen in irradiated normal mice. Thus, the antitumor effect associated with the homeostatic expansion of transfused $\mathrm{T}$ cells in lymphopenic hosts occurs only if the $T$ cells encounter the tumor antigen(s) in LNs. Our observations are compatible with those of Ochsenbein et al. (35), who found that metastasizing tumors that failed to "seed" in LNs and spleen were "ignored" by the immune system. Additionally, no cytotoxic T cells could be induced in tumor cell-challenged alymphoplastic $(A l y \times A l y)$ B6 mice that lack LNs, but possess lymphatic vessels, spleen, and T cells. Antigen presentation at this site may be mediated directly by the tumor cells, by trafficking of tumor antigen-loaded DCs from the tumor site to the draining lymph node, and/or indirectly through cross-priming $(36,37)$.

Remarkably, the antimelanoma effect was associated with long-term specific memory, since rechallenge with the same tumor cells resulted in rejection, while growth of an unrelated tumor (colon carcinoma) was unimpeded. Moreover, homeostatic T cell proliferation was effective even after the melanoma tumor had been established, without the need for tumor cell rechallenge. It is likely that, during the primary tumor cell injection, some of these cells or antigens thereof were transported to the draining LNs, but the response, if any, was ineffective due to the predominance of low-affinity $T$ cells and/or low precursor frequency. In contrast, when the tumor antigens become available to the immune system at the initiation of homeostatic proliferation, selection and expansion of a few high-affinity cells led to strong effector function. Previous studies have shown that only a fraction ( $-30 \%)$ of T cells can undergo homeostatic proliferation (13), and this fraction appears to encompass cells with higher self-affinity (38).

Our findings clearly indicate that $\mathrm{T}$ cells can be induced to mount an effective autoimmune response against self-antigens when homeostatic expansion occurs at the time of antigen encounter. The expanded $\mathrm{T}$ cells should recognize a wide range of self-molecules, but this response was primarily tumor specific, as shown by cytotoxicity and IFN- $\gamma$ induction upon incubation of the expanded cells with the relevant, but not irrelevant, tumor cells. This is probably because the tumor-associated self-antigens are highly enriched in regional LNs. This conclusion is supported by the findings of Oehen and Brduscha-Riem (33), who showed that in vivo antiviral effector function by homeostatically expanding LCMV-specific transgenic T cells depended on the presence of the antigen at the early stages of expansion. The present findings are also in agreement with those of Asavaroengchai et al. (39), who observed efficient antitumor responses in mice immunized with DCs pulsed with tumor cell lysates in the early phase of bone marrow reconstitution, wherein the lymphopenic environment leads to homeostasis-driven T cell proliferation. Preliminary findings by $\mathrm{Hu}$ et al. (40) have also shown efficacy of homeostatic $\mathrm{T}$ cell proliferation in reducing the numbers of pulmonary metastases in mice challenged with a poorly immunogenic prostate tumor.

Several vaccination methods have been shown to overcome tolerance and elicit effective antitumor autoimmune responses, but these labor-intensive procedures are often based on the use of a single epitope that might not induce a broadly efficacious response. In contrast, the procedure described here relies on induction of 
lymphopenia in conjunction with whole tumor cell injection and transfusion of naive, unprimed, autologous $T$ cells with a wide spectrum of self-affinities. This is simple and likely initiates a broad antitumor response without the a priori need to know the tumor antigen(s). Even when specific vaccines are used, it seems advantageous to couple them with simultaneous induction of $\mathrm{T}$ cell homeostatic proliferation, since it is likely to enhance the antitumor response and effectiveness. Furthermore, the present results suggest that, in clinical practice, tumor immunotherapy should commence immediately after completion of irradiation or cytotoxic therapies to take advantage of the lymphopenia and attendant homeostatic $T$ cell expansion.

\section{Acknowledgments}

This is manuscript number 14647IMM from the Department of Immunology, The Scripps Research Institute. The work of the authors reported herein was supported by NIH grants AR-39555, AR-31203, and AG-15061 (A.N. Theofilopoulos), and NIH grant CA-83856 and funds from the Cancer Research Fund under Interagency Agreement 2110020 (University of California Contract 00-0078V, to R.A. Reisfeld). A.G. Niethammer is a fellow of the Deutsche Krebshife.

1. Rosenberg, S.A. 2001. Progress in human tumour immunology and immunotherapy. Nature. 411:380-384.

2. Boon, T., and Old, L.J. 1997. Cancer tumor antigens. Curr. Opin. Immunol. 9:681-683.

3. Pardoll, D.M. 1999. Therapeutic vaccination for cancer. Proc. Natl. Acad. Sci. USA. 96:5340-5342.

4. Nanda, N.K., and Sercarz, E.E. 1995. Induction of anti-self-immunity to cure cancer. Cell. 82:13-17.

5. Houghton, A.N. 1994. Cancer antigens: immune recognition of self and altered self. J. Exp. Med. 180:1-4

6. Houghton, A.N., Gold, J.S., and Blachere, N.E. 2001. Immunity against cancer: lessons learned from melanoma. Curr. Opin. Immunol. 13:134-140.

7. Overwijk, W.W., and Restifo, N.P. 2000. Autoimmunity and the immunotherapy of cancer: targeting the "self" to destroy the "other". Crit. Rev. Immunol. 20:433-450.

8. Gilboa, E. 2001. The risk of autoimmunity associated with tumor immunotherapy. Nat. Immunol. 2:789-792.

9. Gunzer, M., Janich, S., Varga, G., and Grabbe, S. 2001. Dendritic cells and tumor immunity. Semin. Immunol. 13:291-302.

10. Nestle, F.O., Banchereau, J., and Hart, D. 2001. Dendritic cells: on the move from bench to bedside. Nat. Med. 7:761-765.

11. Mackall, C.L., Hakim, F.T., and Gress, R.E. 1997. Restoration of T-cell homeostasis after T-cell depletion. Semin. Immunol. 9:339-346.

12. Goldrath, A.W., and Bevan, M.J. 1999. Selecting and maintaining a diverse T-cell repertoire. Nature. 402:255-262.

13. Surh, C.D., and Sprent, J. 2000. Homeostatic T cell proliferation. How far can T cells be activated to self-ligands? J. Exp. Med. 192:F9-F14.

14. Marrack, P., et al. 2000. Homeostasis of alpha/beta TCR+ T cells. Nat. Immunol. 1:107-111.

15. Theofilopoulos, A.N., Dummer, W., and Kono, D.H. 2001. T cell homeostasis and systemic autoimmunity. J. Clin. Invest. 108:335-340. doi:10.1172/JCI200112173.

16. Udaka, K., Weismuller, H.H., Kienle, S., Jung, G., and Walden, P. 1996. Self-MHC-restricted peptides recognized by an alloreactive T lymphocyte clone. J. Immunol. 157:670-678.
17. Haraguchi, M., et al. 1994. Isolation of GD3 synthase gene by expression cloning of GM3 $\alpha$-2,8-sialtransferase cDNA using anti-CD2 monclonal antibody. Proc. Natl. Acad. Sci. USA. 91:10455-10459.

18. Clarke, P., Mann, J., Simpson, J.F., Ricard-Dickson, K.J., and Primus, F.J. 1998. Mice transgenic for human carcinoembryonic antigen as a model for immunotherapy. Cancer Res. 58:1469-1477.

19. Gavin, M.A., Clarke, S.R., Negrou, A.G., and Rudensky, A. 2002. Homeostasis and anergy of $\mathrm{CD} 4{ }^{+} \mathrm{CD} 25^{+}$suppressor $\mathrm{T}$ cells in vivo. Nat. Immunol. 3:33-41.

20. Dummer, W., Ernst, B., LeRoy, E., Lee, D.S., and Surh, C.D. 2001. Autologous regulation of naive $\mathrm{T}$ cell homeostasis within the $\mathrm{T}$ cell compartment. J. Immunol. 166:2460-2468.

21. Pardoll, D.M., and Topalian, S.L. 1998. The role of CD4+ T cell responses in anti-tumor immunity. Curr. Opin. Immunol. 10:588-594.

22. Sutmuller, R.P.M., et al. 2001. Synergism of cytotoxic T lymphocyte-associated antigen 4 blockage and depletion of CD25+ regulatory $\mathrm{T}$ cells in anti-tumor therapy reveals alternative pathways for suppression of autoreactive cytotoxic T lymphocyte responses. J. Exp. Med. 194:823-832.

23. Overwijk, W.W., et al. 1999. Vaccination with a recombinant vaccinia virus encoding a "self" antigen induces autoimmune vitiligo and tumor cell destruction in mice: requirement for CD4(+) T lymphocytes. Proc. Natl. Acad. Sci. USA. 96:2982-2987.

24. Surman, D.R., Dudley, M.E., Overwijk, W.W., and Restifo, N.P. 2000. Cutting edge: CD4+ T cell control of CD8+ T cell reactivity to a model tumor antigen. J. Immunol. 164:562-565.

25. Trobonjaca, Z., et al. 2001. MHC-II-independent CD4+ T cells induce colitis in immunodeficient RAG-/- hosts. J. Immunol. 166:3804-3812.

26. Corazza, N., Eichenberger, S., Eugster, H.P., and Mueller, C. 1999. Non-lymphocyte-derived tumor necrosis factor is required for induction of colitis in recombination activating gene (RAG)2(-/-) mice upon transfer of CD4(+)CD45RB(hi) T cells. J. Exp. Med. 190:1479-1492.

27. Franco, A., et al. 2000. Epitope affinity for MHC class I determines helper requirement for CTL priming. Nat. Immunol. 1:145-150.

28. Wang, B., et al. 2001. Multiple paths for activation of naïve $\mathrm{CD}^{+} \mathrm{T}$ cells: CD4-independent help. J. Immunol. 167:1283-1289.

29. DeTogni, P., et al. 1994. Abnormal development of peripheral lymphoid organs in mice deficient in lymphotoxin. Science. 264:703-707.

30. Banks, T.A., et al. 1995. Lymphotoxin-alpha-deficient mice: effects on secondary lymphoid organ development and humoral immune responsiveness. J. Immunol. 155:1685-1693.

31. Wagner, N., et al. 1998. L-selectin and $\beta 7$ integrin synergistically mediate lymphocyte migration to mesenteric lymph nodes. Eur. J. Immunol. 28:3832-3839.

32. Murali-Krishna, K., and Ahmed, R. 2000. Cutting edge: naive T cells masquerading as memory cells. J. Immunol. 165:1733-1737.

33. Oehen, S., and Brduscha-Reim, K. 1999. Naive cytotoxic T lymphocytes spontaneously acquire effector function in lymphocytopenic recipients: a pitfall for T cell memory studies? Eur. J. Immunol. 29:608-614.

34. Cho, B.K., Rao, V.P., Ge, Q., Eisen, H.N., and Chen, J. 2000. Homeostasis-stimulated proliferation drives naive $\mathrm{T}$ cells to differentiate directly into memory T cells. J. Exp. Med. 192:549-556.

35. Ochsenbein, A.F., et al. 2001. Roles of tumour localization, second signals and cross priming in cytotoxic T-cell induction. Nature. 411:1058-1064

36. Yewdell, J.W., Norbury, C.C., and Bennink, J.R. 1999. Mechanisms of exogenous antigen presentation by MHC class I molecules in vitro and in vivo: implications for generating $\mathrm{CD} 8+\mathrm{T}$ cell responses to infectious agents, tumors, transplants and vaccines. Adv. Immunol. 73:1-77.

37. Heath, W.R., and Carbone, F.R. 2001. Cross-presentation, dendritic cells, tolerance and immunity. Annu. Rev. Immunol. 19:47-64.

38. Ge, Q., Rao, V.P., Cho, B.K., Eisen, H.N., and Chen, J. 2001. Dependence of lymphopenia-induced $\mathrm{T}$ cell proliferation on the abundance of peptide/MHC epitopes and strength of their interaction with T cell receptors. Proc. Natl. Acad. Sci. USA. 98:1728-1733.

39. Asavaroengchai, W., Kotera, Y., and Mule, J.J. 2002. Tumor lysate-pulsed dendritic cells can elicit an effective antitumor immune response during early lymphoid recovery. Proc. Natl. Acad. Sci. USA. 99:931-936.

40. Hu, H.-M., Urga, W.J., and Fox, B.A. 2001. Homeostasis-driven proliferation sensitizes $\mathrm{T}$ cells to cross-priming by a prostate tumor vaccine. Proc. Am. Assoc. Cancer Res. 42:330. (Abstr.) 